\title{
EDITORIAL
}

\section{Oncology drug pricing structure is broken}

W hen attending the Worldwide Innovative Networking (WIN) conference in Paris at the end of June, a sobering comment made by one presenter, which was echoed by the audience and panellists, was that the biggest toxicity to cancer patients is the cost of cancer treatment-namely, drugs. In the USA, even insured patients can incur out-of-pocket costs of $\$ 70,000$ to $\$ 100,000$. In 2014 , the cost of every new cancer drug approved by the FDA exceeded $\$ 120,000$ per year of use. Some experts have suggested that if something doesn't change-and soon-100\% of the disposable income of US citizens affected by cancer will be spent on treatment costs. Given that one in three people will be diagnosed with cancer in their lifetime, we have a serious problem on our hands. With the costs of some of the newest targeted therapies and immunotherapies being 4,000 times the price of gold, how did we allow such a situation to arise? More crucially, how can we circumvent the mess that the oncology drug development infrastructure finds itself in? This issue is impossible to answer fully without delving into the underlying political and economic reasons. But, regardless of these issues, we know cancer drug costs are not sustainable. Tinkering with the existing system by developing alternative payment models is unlikely to have a notable impact: a drastic overhaul is needed.

The Nature Reviews Clinical Oncology editorial team scans the vast oncology literature each week, and from this assessment trial redundancy is blatantly evident; frequently two or more trials are performed in similar patient cohorts, with both studies asking the same basic research question, and with the same agents being tested. However, quite often these trials do not arrive at the same conclusion or fail to provide a definitive, practicechanging outcome. Since clinical trials cost many millions of monies-a major area of expenditure for industry when bringing new drugs to market-increased efficiency, and probably an altogether new approach, is desperately needed. Moreover, owing to the strict inclusion/exclusion criteria, patient populations in trials often do not reflect 'real world' patients seen in daily clinical practice. As the future of oncology presides around testing drug combinations, this exacerbates the time and money needed to test drugs in the randomized trial setting. To summarize, we have a warped system that is inefficient and allows drugs to be marketed at extortionate prices, often for only a few weeks survival advantage, and in many cases, with increased treatment toxicity to patients.

Although a recent Mayo Clinic Proceedings commentary has highlighted ways that drug pricing could be challenged, including the option for Medicare to negotiate drug prices, what else can be done? In the UK, many of the latest drugs are not approved for use within the NHS, so patients don't even get access to these drugs -irrespective of pricing structure negotiations. Thus, drug costs and drug development are global issues, which will require novel and innovative approaches to be tailored to the very varied needs of health-care systems.

One way to tackle trial redundancy is to establish an independent overarching clinical trials organization to oversee trial design and address the most-appropriate research questions, globally. Such an approach could reduce costs, by limiting trial redundancy or failure, and thereby also the need for reimbursement. In addition, this organization could conduct parallel post-marketing studies and prospective trials to assess the efficacy of drugs in 'real world' patients and compare these outcomes routinely to the RCT data on which the drugs were approved; the savings made by not conducting ineffective trials could be invested into post-marketing studies. Any mismatch in efficacy in post-marketing studies versus the drug-approval trials could help redirect future research questions and trials, and might serve to reset the payment paradigm deemed acceptable to society. Of course, the NCI-MATCH and prospective, nonrandomized TAPUR trials are attempting to achieve this to some degree in the USA, but more needs to be done.

Undeniably, 'me too' drugs involve a lot less preclinical innovation and development costs, so unless nextgeneration agents prove significantly superior to the original drug, this should be reflected in the final price, assuming trial costs are similar. It is a travesty that in low-middle-income countries, trials that could benefit patients, such as those testing metronomic chemotherapy, never materialize because of lack of investment interest. It is wrong that profitability drives many trials. On the other hand, rewarding innovation in situations where some agents outperform expectations, especially if applicable to a broader patient population than hypothesized, should be considered in pricing structures. Importantly, a maximum limit should be set for return of investment from a patient-benefit perspective. How we define QALYs and cost-effectiveness should also be scrutinized. In situations when high-priced drugs do not deliver, there is just cause to re-evaluate the pricing structure, not keep the pricing fixed for decades until patents expire. By considering trial and drug costs globally, we can tackle these issues. We need to act now.

doi:10.1038/nrclinonc.2015.141

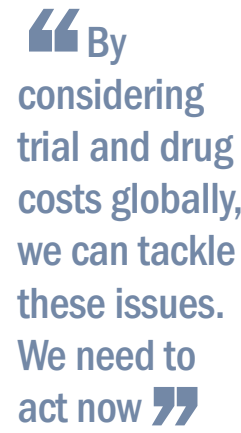

Lisa Hutchinson is the Chief Editor of Nature Reviews Clinical Oncology.

Competing interests The author declares no competing interests. 